\title{
Counting Sums of Two Squares: The Meissel-Lehmer Method
}

\author{
By P. Shiu
}

\begin{abstract}
In 1870, E. D. F. Meissel developed a method for computing the individual values of the prime-counting function, and, in 1959, D. H. Lehmer simplified and extended Meissel's method. Let $W(x)$ count the numbers not exceeding $x$ that are sums of two squares. We develop a variant of the Meissel-Lehmer method for $W(x)$ and use it to calculate $W\left(10^{12}\right)$.
\end{abstract}

1. Introduction. Let $W$ denote the set of positive integers that are sums of two squares. Euler proved that $n \in W$ if and only if every prime divisor $p$ of $n$ satisfying $p \equiv 3(\bmod 4)$ divides $n$ to an exact even power. In 1908, Landau [6] proved that if $W(x)$ counts those $n \in W$ with $n \leqslant x$, then, as $x \rightarrow \infty$,

$$
W(x) \sim B x / \sqrt{\log x},
$$

where

$$
B=\frac{1}{\sqrt{2}} \prod_{p \equiv 3(\bmod 4)}\left(1-\frac{1}{p^{2}}\right)^{-1 / 2} .
$$

However, the exact value for $W(x)$ has not been given for any $x \geqslant 10^{5}$.

Let $U$ be the subset of squarefree odd members of $W$, and let $V$ be the subset of those $n \in W$ with the property that 2 divides $n$ to an exact even power. We observe that every number $n$ can be written uniquely as $l m$, where $l$ is a squarefree odd number and $m$ is either a square or twice a square; and it is clear that $n \in W$ if and only if $l \in U$. Let $U(x)$ and $V(x)$ be the counting functions on the sets $U$ and $V$, respectively. We then have

$$
V(x)=\sum_{k \leqslant \sqrt{x}} U\left(x / k^{2}\right)
$$

and

$$
W(x)=V(x)+V(x / 2) .
$$

It may be of interest to point out that $W(x)$ can be interpreted geometrically as the number of circles centered at the origin, passing through lattice points and having radii not exceeding $\sqrt{x}$. Thus our problem is related to the circle problem of Gauss, which is concerned with the number $R(x)$ of lattice points inside the circle centered

Received October 24, 1984; revised May 3, 1985 and September 20, 1985.

1980 Mathematics Subject Classification. Primary 10J05, $10 \mathrm{H} 30$.

Key' words and phrases. Meissel-Lehmer method, sums of two squares.

(C)1986 American Mathematical Society $0025-5718 / 86 \$ 1.00+\$ .25$ per page 
at the origin with radius $\sqrt{x}$. It is known that $R(x)$ has the asymptotic formula

$$
R(x)=\pi x+O\left(x^{\theta}\right)
$$

where $\frac{1}{4}<\theta<\frac{1}{3}$ (see, for example, [3]) and exact values for $R(x)$, with $x$ up to about $7 \times 10^{10}$, are given in [4].

This paper develops an efficient method for evaluating $U(x)$ so that $W(x)$ can be obtained from (1.3) and (1.4). By Euler's theorem, $U$ is the set of products of distinct primes $p \equiv 1(\bmod 4)$, and so the usual sieve argument, which involves sifting the arithmetic progression $n \equiv 1(\bmod 4), n \leqslant x$, through primes $p \leqslant \sqrt{x}$, will give the simple formula (2.2) for $U(x)$. But this formula is of little practical or theoretical use because of the large number of terms associated with the "Legendre sum". In other words, we encounter the same difficulty as in applying the sieve of EratosthenesLegendre in the evaluation of $\pi(x)$, the number of primes not exceeding $x$. In 1870 Meissel [8] developed a formula for $\pi(x)$ which involves sifting the primes $p \leqslant x^{1 / 3}$ only, and in 1959 Lehmer [7] generalized this formula so that it involves sifting through only the first $a$ primes, where $a$ is a parameter which he set as $\pi\left(x^{1 / 4}\right)$. The purpose of this paper is to develop the Meissel-Lehmer method for the evaluation of $U(x)$, which is then applied to calculate $W(x)$ with $x=10^{k}$ for $k=1,2, \ldots, 12$.

Very recently, Lagarias, Miller and Odlyzko [5] gave further refinements of the Meissel-Lehmer method for $\pi(x)$ and gave an asymptotic running time analysis of their algorithm on a Random Access Machine with and without parallel processors. Much of what they discussed is, of course, very relevant to the problem discussed here. The author is indebted to the referee for mentioning their work and is grateful to the editor for a preprint of [5].

2. Notation. The letters $p, q, r$ are reserved for primes with $q \equiv 1(\bmod 4), r \equiv 3$ $(\bmod 4)$. We write $p(0)=2$, and, for $a \geqslant 1, p(a)$ denotes the $a$ th odd prime; also,

$$
p(a, b)=p(a) p(a+1) \cdots p(b), \quad 1 \leqslant a<b .
$$

For an odd squarefree integer $d$, we define $\delta=\delta(d)$ by $\delta=d \Pi_{q \mid d} q$; that is, if $d=\alpha \beta$ where $\alpha, \beta$ are products of primes $q, r$ respectively, then $\delta=\alpha^{2} \beta$. As usual, $\mu(d)$ is the Möbius function and $\chi(d)$ is the nonprincipal character mod 4 , and we shall write $\delta_{a}$ and $\chi_{a}$ for $\delta((p(a))$ and $\chi(p(a))$, respectively.

We next define, for odd $l$,

$$
N(x ; d, l)=|\{n: 1 \leqslant n \leqslant x, \delta(d) \mid n, n \equiv l(\bmod 4)\}|,
$$

and

$$
\varphi(x ; a, l)=\sum_{d \mid p(1, a)} \mu(d) N(x ; d, l) .
$$

We call $\phi(x ; a, l)$ a Legendre sum; by the inclusion-exclusion principle it counts the numbers $n \leqslant x, n \equiv l(\bmod 4)$ with the property that $n$ is not divisible by $q^{2}$, nor by $r$, for any $q, r \leqslant p(a)$. In particular, therefore,

$$
\varphi(x ; a, 1)=U(x) \text { if } p(a+1)>x^{1 / 2} .
$$

By the Meissel-Lehmer formula for $U(x)$, we mean an extension of this formula relating $U(x)$ and the Legendre sum $\varphi(x ; a, 1)$, which holds when $p(a+1) \leqslant x^{1 / 2}$. 
The numbers of primes $p, r \leqslant x$ are denoted by $\pi(x)$ and $\pi_{4,3}(x)$, respectively, and the $m$ th number in $U$ is denoted by $u_{m}$. For fixed $x$ and $a$ with $p(a)<\sqrt{x}$ we can choose $M$ so that $1 \leqslant u_{M}<x / p^{2}(a)$ and we define

$$
x_{m}=\sqrt{x / u_{m}}, \quad 1 \leqslant m \leqslant M,
$$

so that $p(a)<x_{M}<\cdots<x_{1}=\sqrt{x}$. In Section 5 we shall also write $x_{M+1}$ for $p(a)$.

3. The Meissel-Lehmer Formula for $U(x)$. We need some more notation in order to state the Meissel-Lehmer formula for $U(x)$. Let $k=k(n, p)$ denote the highest power of $p$ which divides $n$, that is $p^{k} \mid n$ and $p^{k+1}+n$. We define

$$
\Omega(n ; a, b)=2 \sum_{p(a)<q \leqslant p(b)}[k(n, q) / 2]+\sum_{p(a)<r \leqslant p(b)} k(n, r) .
$$

In other words, $\Omega(n ; a, b)$ counts the prime divisors $q, r$ of $n$ with $p(a)<q$, $r \leqslant p(b)$, taking account of multiplicity in such a way that each $q$ is counted only the greatest even number of times. With this notation, we see that $U$ is the set of odd numbers $n$ with $\Omega(n ; 0, n)=0$.

For $k \geqslant 1$, we now define $U_{k}(x, a)$ to be the number of odd numbers $n \leqslant x$ with

$$
\Omega(n ; 0, a)=0, \quad \Omega(n ; a, n)=k .
$$

For example, $U_{2}(x, a)$ counts the odd numbers $n \leqslant x$ which are not divisible by $q^{2}$, nor by $r$, for any $q, r \leqslant p(a)$, but are either divisible by $q^{2}$ (and possibly $q^{3}$, but not $q^{4}$ ) for precisely one $q>p(a)$, or divisible by $r_{1} r_{2}$ for precisely two $r_{1}, r_{2}$ with $r_{2} \geqslant r_{1}>p(a)$, but not divisible by $q^{2} r$ for any $q, r>p(a)$. The Meissel-Lehmer formula for $U(x)$ now takes the form

$$
\varphi(x ; a, 1)=U(x)+U_{2}(x, a)+U_{4}(x, a)+\cdots,
$$

and we also have

$$
\varphi(x ; a,-1)=U_{1}(x, a)+U_{3}(x, a)+U_{5}(x, a)+\cdots .
$$

Both these series terminate since $U_{k}(x, a)=0$ if $p(a+1)>x^{1 / k}$. In particular, therefore,

$$
\varphi(x ; a, 1)=U(x)+U_{2}(x, a) \text { if } p(a+1)>x^{1 / 4},
$$

and

$$
\varphi(x ; a,-1)=U_{1}(x) \text { if } p(a+1)>x^{1 / 3} .
$$

Our next task is to develop methods, which are parallel to those of Meissel and Lehmer, to evaluate $\varphi(x ; a, l)$ and $U_{2}(x, a)$ so that $U(x)$ can be obtained from (3.1).

For fixed $x$, the computing times of $\varphi(x ; a, 1)$ and $U_{2}(x, a)$ are increasing and decreasing functions of $a$, respectively. In practice we choose $a$ so that the computing times are about the same, and we check the calculations by recomputing $U(x)$ with a small change in the value of $a$.

4. The Buchstab Iteration Formula for $\varphi(x ; a, l)$. The sieve formula $(2.2)$ is, of course, only a special case of a combinatorial identity arising from the inclusionexclusion principle. There is a simple iteration formula associated with the Legendre sum of the problem, and following Halberstam and Richert [1] we name such an iteration formula after Buchstab who was the first to apply this type of iteration formula fruitfully in sieve methods. For our Legendre sum the Buchstab iteration 
formula takes the form: For $1 \leqslant a<b$,

$$
\varphi(x ; b, l)=\sum_{d \mid p(a+1, b)} \mu(d) \varphi\left(\frac{x}{\delta(d)} ; a, \chi(d) l\right) .
$$

The proof of this is rather simple. Both $\delta(d)$ and $\chi(d)$ are multiplicative functions and we always have $\delta(d) \chi(d) \equiv 1(\bmod 4)$. Therefore, for coprime squarefree odd $d_{1}, d_{2}$, we have

$$
\begin{aligned}
& N\left(x ; d_{1} d_{2}, l\right)= \sum_{\substack{n \leqslant x \\
\delta\left(d_{1} d_{2}\right) \mid n \\
n \equiv l(\bmod 4)}} 1=\sum_{\substack{m \leqslant x / \delta\left(d_{2}\right) \\
\delta\left(d_{1}\right) \mid m \\
m \equiv \chi\left(d_{2}\right) l(\bmod 4)}} 1 \\
&=N\left(\frac{x}{\delta\left(d_{2}\right)} ; d_{1}, \chi\left(d_{2}\right) l\right)
\end{aligned}
$$

Next, if $d \mid p(1, b)$, then $d=d_{1} d_{2}$ where $d_{1}\left|p(1, a), d_{2}\right| p(a+1, b)$, so that

$$
\begin{aligned}
\varphi(x ; b, l) & =\sum_{d_{1} \mid p(1, a)} \sum_{d_{2} \mid p(a+1, b)} \mu\left(d_{1}\right) \mu\left(d_{2}\right) N\left(x ; d_{1} d_{2}, l\right) \\
& =\sum_{d_{2} \mid p(a+1, b)} \mu\left(d_{2}\right) \sum_{d_{1} \mid p(1, a)} \mu\left(d_{1}\right) N\left(\frac{x}{\delta\left(d_{2}\right)} ; d_{1}, \chi\left(d_{2}\right) l\right) \\
& =\sum_{d_{2} \mid p(a+1, b)} \mu\left(d_{2}\right) \varphi\left(\frac{x}{\delta\left(d_{2}\right)} ; a, \chi\left(d_{2}\right) l\right),
\end{aligned}
$$

which proves (4.1).

On replacing $a$ with $a-1$ and setting $b=a$ in (4.1), we have

$$
\varphi(x ; a, l)=\varphi(x ; a-1, l)-\varphi\left(\frac{x}{\delta_{a}} ; a-1, \chi_{a} l\right)
$$

and repeated application of this gives: For $1 \leqslant a<a^{\prime}$,

$$
\varphi\left(x ; a^{\prime}, l\right)=\varphi(x ; a, l)-\sum_{a<h \leqslant a^{\prime}} \varphi\left(\frac{x}{\delta_{h}} ; h-1, \chi_{h} l\right) .
$$

5. The Computation of $U_{2}(x, a)$. Every $U_{k}(x, a)$ can be expressed as a $k$-fold sum $\Sigma U\left(x / p_{1} \cdots p_{k}\right)$ over the primes $p_{1}, \ldots, p_{k}$. For example, since each number counted by $U_{1}(x, a)$ has the form $r n$ where $r>p(a), n \in U, n \leqslant x / r$, it follows that

$$
U_{1}(x, a)=\sum_{r>p(a)} U(x / r)
$$

Similarly, we have $U_{2}(x, a)=S(x, a)+T(x, a)$, where

$$
S(x, a)=\sum_{p>p(a)} U\left(x / p^{2}\right), \quad T(x, a)=\sum_{r_{2}>r_{1}>p(a)} U\left(x / r_{1} r_{2}\right) .
$$

The main problem lies with the double sum $T(x, a)$ which can be interpreted geometrically as the total weight of the lattice points $\left(r_{1}, r_{2}\right)$ in the region $p(a)<r_{1}$ $<r_{2}, r_{1} r_{2} \leqslant x$, where each point is given the weight $m$ if $x / u_{m+1}<r_{1} r_{2} \leqslant x / u_{m}$. Observe that the upper limit for $r_{2}$ in the double sum $T(x, a)$ is $x / p(a)$, and when this is large, a direct evaluation of $T(x, a)$ is difficult. We introduce a parameter $M$ whereby we shall make a direct evaluation of the contribution from those points with $r_{1} r_{2} \leqslant x / u_{M}$. The remaining points satisfy $x / u_{M}<r_{1} r_{2} \leqslant x$, and these are considered as a counting problem so that their contribution is rewritten as various sums involving the $r$-prime counting function $\pi_{4,3}(z)$. More precisely, we have the 
following

LEMMA. Let $1 \leqslant u_{M}<x / p^{2}(a)$. Then

where

$$
T(x, a)=T_{1}(x ; a, M)+T_{2}(x ; a, M),
$$

$$
\begin{gathered}
T_{1}(x ; a, M)=\sum_{x_{M+1}<r_{1} \leqslant x_{M}} \sum_{r_{1}<r_{2} \leqslant x / r_{1} u_{M}}\left(U\left(\frac{x}{r_{1} r_{2}}\right)-M\right), \\
T_{2}(x ; a, M)=\sum_{1 \leqslant m \leqslant M}\left(\sum_{x_{m+1}<r \leqslant x_{m}} \sum_{1 \leqslant l \leqslant m} \pi_{4,3}\left(\frac{x}{r u_{l}}\right)-f\left(x_{m}\right)\right)+M f\left(x_{M+1}\right),
\end{gathered}
$$

and

$$
\begin{gathered}
x_{m}=\sqrt{\frac{x}{u_{m}}}, \quad m=1,2, \ldots, M ; \quad x_{M+1}=p(a), \\
f(z)=\frac{1}{2} \pi_{4,3}(z)\left(\pi_{4,3}(z)+1\right) .
\end{gathered}
$$

Proof. Let us write

$$
R(x ; A, B)=\sum_{A<r_{1} \leqslant B} \sum_{r_{1}<r_{2} \leqslant x / r_{1}} U\left(\frac{x}{r_{1} r_{2}}\right),
$$

so that

$$
T(x, a)=R(x ; p(a), \sqrt{x})=R\left(x ; x_{M+1}, x_{M}\right)+R\left(x ; x_{M}, x_{1}\right) .
$$

We first consider

$$
R\left(x ; x_{M+1}, x_{M}\right)=\sum_{x_{M+1}<r_{1} \leqslant x_{M}}\left\{\sum_{r_{1}<r_{2} \leqslant x / r_{1} u_{M}} U\left(\frac{x}{r_{1} r_{2}}\right)+\sum_{x / r_{1} u_{M}<r_{2} \leqslant x / r_{1}} U\left(\frac{x}{r_{1} r_{2}}\right)\right\},
$$

and we rewrite the second inner sum as

$$
\sum_{1 \leqslant m<M} \sum_{x / r_{1} u_{m+1}<r_{2} \leqslant x / r_{1} u_{m}} U\left(\frac{x}{r_{1} r_{2}}\right) .
$$

But if $u_{m} \leqslant x / r_{1} r_{2}<u_{m+1}$, then $U\left(x / r_{1} r_{2}\right)=m$, so that this sum becomes

$$
\sum_{1 \leqslant m<M} m\left(\pi_{4,3}\left(\frac{x}{r_{1} u_{m}}\right)-\pi_{4,3}\left(\frac{x}{r_{1} u_{m+1}}\right)\right)=\sum_{1 \leqslant m \leqslant M} \pi_{4,3}\left(\frac{x}{r_{1} u_{m}}\right)-M r_{4,3}\left(\frac{x}{r_{1} u_{M}}\right) \text {. }
$$

From

$$
\begin{aligned}
\sum_{x_{M+1}<r_{1} \leqslant x_{M}} \sum_{r_{1}<r_{2} \leqslant x / r_{1} u_{M}} 1 & =\sum_{x_{M+1}<r_{1} \leqslant x_{M}}\left(\pi_{4,3}\left(\frac{x}{r_{1} u_{M}}\right)-\pi_{4,3}\left(r_{1}\right)\right) \\
& =\sum_{x_{M+1}<r_{1} \leqslant x_{M}} \pi_{4,3}\left(\frac{x}{r_{1} u_{M}}\right)-\sum_{\pi_{4,3}\left(x_{M+1}\right)<i \leqslant \pi_{4,3}\left(x_{M}\right)} i \\
& =\sum_{x_{M+1}<r_{1} \leqslant x_{M}} \pi_{4,3}\left(\frac{x}{r_{1} u_{M}}\right)-f\left(x_{M}\right)+f\left(x_{M+1}\right),
\end{aligned}
$$

it now follows that

$$
\begin{aligned}
R\left(x ; x_{M+1}, x_{M}\right)= & T_{1}(x ; a, M) \\
& +\sum_{x_{M+1}<r \leqslant x_{M}} \sum_{1 \leqslant m \leqslant M} \pi_{4,3}\left(\frac{x}{r u_{m}}\right)-m\left(f\left(x_{m}\right)-f\left(x_{m+1}\right)\right) .
\end{aligned}
$$

Similarly we have, for $1 \leqslant m<M$,

$$
R\left(x ; x_{m+1}, x_{m}\right)=\sum_{x_{m+1}<r \leqslant x_{m}} \sum_{1 \leqslant l \leqslant m} \pi_{4,3}\left(\frac{x}{r u_{m}}\right)-m\left(f\left(x_{m}\right)-f\left(x_{m+1}\right)\right),
$$


and the required result follows from

$$
R\left(x ; x_{M}, x_{1}\right)=\sum_{1 \leqslant m<M} R\left(x ; x_{m+1}, x_{M}\right) .
$$

The evaluation of $T(x, a)$ is similar to, but more complicated than, that for $P_{2}(x, a)$ in the prime counting problem. We first encode the primes $p, r$ in $2 \leqslant p$ $\leqslant \sqrt{x / p(a)}$ and $3 \leqslant r \leqslant \sqrt{x}$. For the computation of $T_{1}(x ; a, M)$, we also build a table of values for $U(4 n-3)$ up to $x / p^{2}(a)$, and we encode the numbers $\left[x_{m}\right]$, $1 \leqslant m \leqslant M$, where $M$ is chosen so large that $x_{M} \leqslant \sqrt{x_{1} p(a)}$; this ensures that the upper bound for $r_{2}$ in $T_{1}(x ; a, M)$ is at most $\sqrt{x}$, and the sum can now be evaluated from the table of values $U(4 n-3)$. For the computation of $T_{2}(x ; a, M)$, we build a table of values for $\pi_{4,3}(4 n-1)$ up to $x / p(a)$. This can be divided into blocks of a suitable length so that the values can be encoded into the fast memory of the machine as described in [5]. The sum $T_{2}(x ; a, M)$ can now be evaluated by running this table for $\pi_{4,3}(4 n-1)$ through the individual blocks and reading off the relevant values corresponding to the various points $z=x / r u_{l}$.

The sum $S(x, a)$ can be dealt with similarly and we have

$$
S(x, a)=\sum_{p(a)<p \leqslant x_{M}}\left(U\left(\frac{x}{p^{2}}\right)-M\right)+\sum_{1 \leqslant m \leqslant M} \pi\left(x_{m}\right)-M(a+1) .
$$

6. The Computation of $\varphi(x ; a, l)$. We first describe a simple method of computing the Legendre sum $\varphi(x ; a, l)$. We apply the Buchstab iteration formula (4.1) in the form

where

$$
\begin{aligned}
\varphi(x ; a, l) & =\sum_{d \mid p(5, a)} \mu(d) \varphi\left(\frac{x}{\delta(d)} ; 4, \chi(d) l\right) \\
& =F_{0}-F_{1}+F_{2}-\cdots+(-1)^{K} F_{K}
\end{aligned}
$$

$$
\begin{gathered}
F_{0}=\varphi(x ; 4, l), \\
F_{k}=\sum_{11<p<\cdots<p_{k} \leqslant p(a)} \varphi\left(\frac{x}{\delta\left(p_{1} \cdots p_{k}\right)} ; 4, \chi\left(p_{1} \cdots p_{k}\right) l\right) .
\end{gathered}
$$

The number $K$ is the least integer $L$ with the property that any squarefree odd $d$ with $L+1$ distinct prime divisors $p>11$ must satisfy $\delta(d)>x$. The size of $K$ is rather modest; for example, $K=5,6,6,7$ correspond to $x=10^{9}, 10^{10}, 10^{11}, 10^{12}$.

Since

$$
\delta(p(1,4))=3 \cdot 5^{2} \cdot 7 \cdot 11=5775, \quad \varphi(5775 ; 4, \pm 1)=720,
$$

it follows from periodicity with period $4 \cdot 5775=23100$ that

$$
\varphi(z ; 4, \pm 1)=2880\left(\frac{z}{23100}\right)+\varphi(m ; 4, \pm 1), \quad z>0,
$$

where $m$ is the remainder of $[z]$ when divided by 23100 . If we encode two tables of values for $\varphi(4 n-3 ; 4,1)$ and $\varphi(4 n-1 ; 4,-1)(1 \leqslant 4 n-3<4 n-1 \leqslant 23100)$, then $\varphi(z ; 4, \pm 1)$ can be read off and the Legendre sum $\varphi(x ; a, l)$ can now be evaluated by nesting all the $F_{k}$ into a single program.

When $x$ is large and $a$ is not small (say $x \geqslant 10^{12}, a \geqslant 500$ ), the evaluation of $\varphi(x ; a, l)$ by the above method is very time-consuming. In this case, we should use the refinement of the Meissel-Lehmer method proposed by Lagarias, Miller and 
Odlyzko [5] for the prime counting problem. Their method may be described as a sophisticated application of the formulas (4.1) and (4.2), with a good truncation rule which separates the terms into "ordinary" and "special" leaves of a binary tree developed from (4.1). The simple method described above corresponds to having a truncation rule in which every leaf becomes an ordinary leaf with " $k=4$ ". There is, of course, a similar good truncation rule which will speed up the calculations by having special leaves for $\varphi(x ; a, l)$, but the algorithm for the contribution of these leaves will be even more complicated than that for $\varphi(x, a)$ in the prime counting problem.

7. The Computation of $W(x)$. Since $W(x)$ is given by (1.4), we need only describe the evaluation of $V(x)$ given by (1.3). The sum (1.3) is similar to the sum $S(x, a)$ except that $k$ is not restricted to primes $p>p(a)$. We introduce two parameters $K$, $M$ where $1 \leqslant K \leqslant x_{M}$. Then, as in Section 5 we find that

$$
V(x)=\sum_{1 \leqslant k \leqslant K} U\left(\frac{x}{k^{2}}\right)+\sum_{K<k \leqslant x_{M}}\left(U\left(\frac{x}{k^{2}}\right)-M\right)+\sum_{1 \leqslant m \leqslant M}\left[x_{m}\right]-M K .
$$

In the first sum here, every term $U(z)$ has $z \geqslant x / K^{2}$, and each term has to be evaluated by the method described earlier. The second sum is evaluated in the same way as the first sum for $S(x, a)$; that is, we encode $U(4 n-3)$ for $u_{M} \leqslant 4 n-3<$ $x / K^{2}$ and then run $k$ backwards in $K<k \leqslant x_{M}$ and read off the values for $U\left(x / k^{2}\right)$.

Again $K$ and $M$ can be chosen to minimize the computing time for $V(x)$, but in practice we set

$$
K=\left[\sqrt{x} / 10^{4}\right], \quad M=U\left(x^{1 / 6}\right)
$$

so that we need to evaluate $U(z)$ individually when $z>10^{8}$, and simply read off $U(z)$ from a table of values when $z \leqslant 10^{8}$.

8. Results. We first give an accurate evaluation of Landau's constant $B$ in (1.2). For this purpose, we need to speed up the convergence of the product as follows. We write

$$
4 B^{4}=\prod_{r}\left(1-\frac{1}{r^{2}}\right)^{-2}=\prod_{r}\left(1-\frac{1}{r^{4}}\right)^{-1}\left(1-\frac{1}{r^{2}}\right)^{-1}\left(1+\frac{1}{r^{2}}\right),
$$

and observe that

$$
\prod_{r}\left(1-\frac{1}{r^{2}}\right)^{-1}\left(1+\frac{1}{r^{2}}\right)=\prod_{p>2}\left(1-\frac{1}{p^{2}}\right)^{-1}\left(1-\frac{\chi(p)}{p^{2}}\right) .
$$

Furthermore, the right-hand side here is the Euler product for

$$
\left(1-1 / 2^{2}\right) \zeta(2) / L(2, \chi),
$$

where

$$
\zeta(2)=\sum_{n=1}^{\infty} \frac{1}{n^{2}}=\frac{\pi^{2}}{6}
$$


and

$$
L(2, \chi)=\sum_{n=1}^{\infty} \frac{\chi(n)}{n^{2}}=\sum_{m=0}^{\infty}\left(\frac{1}{(4 m+1)^{2}}-\frac{1}{(4 m+3)^{2}}\right)
$$

Therefore,

$$
32 L(2, \chi) B^{4}=\pi^{2} \prod_{r}\left(1-\frac{1}{r^{4}}\right)^{-1} .
$$

The infinite product here converges much more rapidly and $L(2, \chi)$ can be calculated accurately from the Euler-Maclaurin summation formula. Thus, on listing the 4808 primes $r<10^{5}$ we find that

$$
B=0.764223653589220 \ldots
$$

D. R. Heath-Brown (private communication) has pointed out that one has

$$
2 B^{2}=\prod_{m=1}^{\infty}\left(\frac{\zeta\left(2^{m}\right)}{L\left(2^{m}, \chi\right)}\left(1-2^{-2^{m}}\right)\right)^{2^{-m}}
$$

so that $B$ can be computed without any listing of the primes at all.

We now write $\hat{W}(x), \hat{U}(x), \hat{V}(x)$ for

$$
\frac{B x}{\sqrt{\log x}}, \quad \frac{4 B x}{\pi^{2} \sqrt{\log x}}, \quad-\frac{2 B x}{3 \sqrt{\log x}},
$$

respectively so that, from (1.1), (1.3) and (1.4), as $x \rightarrow \infty$,

$$
W(x) \sim \hat{W}(x), \quad U(x) \sim \hat{U}(x), \quad V(x) \sim \hat{V}(x) .
$$

We give the values of $W(x), U(x), V(x)$ and $\hat{W}(x), \hat{U}(x), \hat{V}(x)$ in the following tables.

TABLE 1

\begin{tabular}{|l|r|r|r|}
\hline$x$ & \multicolumn{1}{|r|}{$W(x)$} & $\hat{W}(x)$ & $\hat{W}(x) / W(x)$ \\
\hline 10 & 7 & 5.40 & 0.7195 \\
$10^{2}$ & 43 & 35.61 & 0.8282 \\
$10^{3}$ & 330 & 290.77 & 0.8811 \\
$10^{4}$ & 2749 & 2518.16 & 0.9160 \\
$10^{5}$ & 24028 & 22523.07 & 0.9374 \\
$10^{6}$ & 216341 & 205606.58 & 0.9504 \\
$10^{7}$ & 1985459 & 1903547.03 & 0.9587 \\
$10^{8}$ & 18457847 & 17806052.01 & 0.9647 \\
$10^{9}$ & 173229058 & 167877068.32 & 0.9691 \\
$10^{10}$ & 1637624156 & 1592621708.36 & 0.9725 \\
$10^{11}$ & 15570523346 & 15185052177.44 & 0.9752 \\
$10^{12}$ & 148736629005 & 145385805873.79 & 0.9775 \\
\hline
\end{tabular}


TABLE 2

\begin{tabular}{|l|r|r|r|}
\hline$x$ & $U(x)$ & $\hat{U}$ & $\hat{U}(x)$ \\
\hline 10 & 2 & 2.04 & 1.0206 \\
$10^{2}$ & 14 & 14.43 & 1.0309 \\
$10^{3}$ & 112 & 117.85 & 1.0522 \\
$10^{4}$ & 997 & 1020.57 & 1.0236 \\
$10^{5}$ & 8982 & 9128.26 & 1.0163 \\
$10^{6}$ & 82305 & 83329.21 & 1.0124 \\
$10^{7}$ & 746092 & 771478.55 & 1.0097 \\
$10^{8}$ & 7159654 & 7216521.06 & 1.0079 \\
$10^{9}$ & 67581778 & 68038013.07 & 1.0068 \\
$10^{10}$ & 641696858 & 645465266.34 & 1.0059 \\
$10^{11}$ & 6112456540 & 6154269841.16 & 1.0068 \\
$10^{12}$ & 58649349743 & 58922647743.71 & 1.0047 \\
\hline
\end{tabular}

TABLE 3

\begin{tabular}{|l|r|r|r|}
\hline$x$ & \multicolumn{1}{|r|}{$V(x)$} & $\hat{V}(x)$ & $\hat{V}(x) / V(x)$ \\
\hline 10 & 4 & 3.36 & 0.8394 \\
$10^{2}$ & 28 & 23.74 & 0.8479 \\
$10^{3}$ & 214 & 193.85 & 0.9058 \\
$10^{4}$ & 1803 & 1678.77 & 0.9311 \\
$10^{5}$ & 15830 & 15015.38 & 0.9485 \\
$10^{6}$ & 142844 & 137071.05 & 0.9596 \\
$10^{7}$ & 1313047 & 1269031.35 & 0.9665 \\
$10^{8}$ & 12220699 & 11870701.34 & 0.9714 \\
$10^{9}$ & 114790260 & 111918045.54 & 0.9750 \\
$10^{10}$ & 1085885280 & 1061747805.58 & 0.9778 \\
$10^{11}$ & 10330026070 & 10123368118.30 & 0.9800 \\
$10^{12}$ & 98719755928 & 96923870582.53 & 0.9818 \\
\hline
\end{tabular}

It will be observed that the error term for $W(x)$ is much bigger than the corresponding result in the prime counting problem. In fact, if the Riemann hypothesis is true, then the error term for $\pi(x)$ is only $O\left(x^{1 / 2} \log x\right)$ and in any case, Littlewood proved that this error term changes sign infinitely often so that it can be small for large $x$. On the other hand, we know that the error term for $W(x)$ is $\Omega_{+}\left(x /(\log x)^{3 / 2}\right)$. In fact (see [2, p. 63]), Landau's asymptotic formula has been improved to the following: For any fixed integer $N \geqslant 1$, as $x \rightarrow \infty$,

$$
W(x)=\sum_{j=1}^{N} B_{j} x(\log x)^{1 / 2-j}+O_{N}\left(x(\log x)^{-N-1 / 2}\right),
$$

where $B_{j}$ are computable constants.

We conclude with the following remarks on the actual computations performed on the Honeywell Multics System at Loughborough University Computing Centre in July 1984. 
(i) The author is a tyro on computations by machines; he can only write programs in FORTRAN, perhaps the least suitable language for the problem. Thus the largest integer allowed by the compiler is $2^{35}-1<4 \times 10^{10}$ and a rather ad hoc and clumsy multi-length arithmetic procedure has to be incorporated into the programs.

(ii) As remarked by Lehmer [7], and shown in [5], the Meissel-Lehmer method is a good example of substituting time for storage allocations. We chose to have the modest size of only 60000 stores and the approximate computing times for $W\left(10^{10}\right)$, $W\left(10^{11}\right)$ and $W\left(10^{12}\right)$ are 15 minutes, 2 hours and 19 hours respectively. Each entry for $U(x), V(x)$ and $W(x)$ has been double checked by varying the parameters $a, M$ and $K$ in the associated formulas.

Department of Mathematics

University of Technology

Loughborough, Leicestershire

England

1. H. Halberstam \& H. E. Richert, Sieve Methods, Academic Press, London, 1974.

2. G. H. HARDY, Ramanujan. Twelve Lectures on Subjects Suggested by His Life and Work, Cambridge Univ. Press, 1940.

3. L. K. HuA, "The lattice-points in a circle," Quart. J. Math. Oxford, v. 13, 1942, pp. 18-29.

4. H. B. Keller \& J. R. Swenson, "Experiments on the lattice problem of Gauss," Math. Comp., v. 17, 1963, pp. 223-230.

5. J. C. Lagarias, V. S. Miller \& A. M. Odlyzko, "Computing $\pi(x)$ : The Meissel-Lehmer method," Math. Comp., v. 44, 1985, pp. 537-560.

6. E. LANDAU, "Über die Einteilung der positiven ganzen Zahlen in vier Klassen nach der Mindestzahl der zu ihrer additiven Zusammensetzung erforderlichen Quadrate," Arch. Math. Phys. (3), v. 13 , 1908, pp. 305-312.

7. D. H. LehMER, “On the exact number of primes less than a given limit," Illinois J. Math., v. 3, 1959 , pp. 381-388.

8. E. D. F. Meissel, “Über die Bestimmung der Primzahlmenge innerhalb gegebener Grenzen,” Math. Ann., v. 2, 1870, pp. 636-642.

Editorial Note. The reader should also consult D. Shanks, "The second-order term in the asymptotic expansion of $B(x), "$ this journal, v. 18, 1964, pp. 75-86, in particular, Eq. (16) on p. 78, Eq. (5') on p. 79. and Table 1 in this reference. 